\title{
Targeting Atrial Fibrillation Patients for Left Atrial Appendage Occlusion with Epicardial Closing Devices
}

\author{
Harry J Crijns, ${ }^{1}$ Stefan G Spitzer ${ }^{2,3}$ and Claudio Tondo ${ }^{4}$
}

1. Maastricht University Medical Center, Maastricht, The Netherlands; 2. Dresden Heart and Vessels Clinic, Dresden, Germany; 3.Brandenburg University of Technology Cottbus-Senftenberg, Institute of Medical Technology, Dresden, Germany; 4. Heart Rhythm Center, Monzino Cardiac Center, Scientific Institute for Research and Healthcare (IRCCS), Deptartment of Clinical Sciences and Community, University of Milan, Milan, Italy

$\mathrm{T}$ he left atrial appendage (LAA) is a key source of thrombi in patients with non-valvular atrial fibrillation (AF) and a major clinical focus for stroke prevention. Current surgical exclusion remains suboptimal and percutaneous approaches targeting the LAA, although well validated, fail to offer complete and durable occlusion for all patients. The high anatomical variability of the LAA demands a more tailored and targeted treatment approach. Epicardial LAA clip occlusion represents an important alternative approach for patients with AF not amenable to catheter closure or where anticoagulation is contraindicated. In this article, we briefly review the clinical evidence for epicardial devices in LAA occlusion, with a focus on the Atriclip and discuss their role in stroke prevention strategies.

\section{Keywords}

Atrial fibrillation, left atrial appendage, Atriclip

Disclosures: Harry J Crijns discloses grants from the institutions CARIM/Maastricht University and Maastricht University Medical Centre, Maastricht, The Netherlands, from Bayer, Bristol-Myers Squibb, Pfizer,

Boehringer-Ingelheim, Daiichi-Sankyo and Medtronic.

Stefan G Spitzer has received speaker's fees and travel

support from Medtronic, St. Jude Medical, Abbott, Topera,

Recordati Pharma and AstraZeneca, and participated

in clinical trials for Medtronic, St. Jude Medical, Servier,

Daiichi Sankyo and Bristol- Myers Squibb. Claudio

Tondo has received speaker's fees and grants from

St. Jude Medical, Abbott, Medtronic, Boston Scientific

and Biosense-Webster. He serves on the EU Medtronic

Advisory Board and the Boston Scientific Advisory Board.

Acknowledgements: Medical writing support

was provided by Helen Boreham, Freelance

Medical Writer, and supported by Atricure.

Review Process: Double-blind peer review.

Compliance with Ethics: This study involves

a review of the literature and did not involve

any studies with human or animal subjects

performed by any of the authors.

Authorship: All named authors meet the International Committee of Medical Journal Editors (ICMJE) criteria

for authorship of this manuscript, take responsibility

for the integrity of the work as a whole, and have given final approval to the version to be published.

open Access: This article is published under the Creative Commons Attribution Noncommercial License, which permits any non-commercial use distribution, adaptation and reproduction provided the original author(s) and source are given appropriate credit. (C) The Authors 2018.

Received: 7 February 2018

Accepted: 26 February 2018

Citation: European Journal of Arrhythmia

\& Electrophysiology, 2018;4(2):53-5

Corresponding Author: Harry J Crijns, Maastricht UMC, P. Debyelaan 25, 6229 HX, PO Box 5800, 6202 AZ Maastricht, The Netherlands. E: hjgm.crijns@mumc.nl

Support: The publication of this article was supported by Atricure.
The left atrial appendage (LAA) is now recognised as a key site of thromboembolic origin in atrial fibrillation (AF), acting as the primary source of around $90 \%$ of embolic strokes in patients with non-valvular $\mathrm{AF}^{1-4} \mathrm{An}$ important therapeutic strategy for stroke prevention in AF targets the LAA and simultaneously offers the opportunity to circumvent long-term oral anticoagulants (OACS).,5 The 2016 European Society of Cardiology (ESC) guidelines on AF management recommend that interventional LAA occlusion may be considered in patients at high stroke risk with contraindications to long-term OACs (Class IIb, level of evidence B). ${ }^{6}$ Surgical LAA occlusion or exclusion is also endorsed (Class IIb, level of evidence B) by the ESC for patients undergoing open-heart surgery or thoracoscopic AF surgery. ${ }^{6}$

\section{Unmet needs in left atrial appendage occlusion}

Approaches targeting occlusion of the LAA in non-valvular AF include surgical exclusion or occlusion, percutaneous catheter-based approaches and minimally invasive epicardial methods. To date, surgical LAA occlusion approaches such as simple suture ligation, endo- or epicardial oversewing and stapling exclusion have yielded disappointing results. ${ }^{5,7}$ In the majority of studies, surgical LAA techniques failed to demonstrate efficacy in stroke prevention and outcomes proved inconclusive, heterogenous or frankly negative as complete occlusion was not achieved.., 7 Undoubtedly, the most important reason for the lack of evidence supporting the various LAA occlusion techniques lies in the small number of patients studied and flawed study methods employed.

Percutaneous LAA closure devices provided the initial clinical evidence supporting LAA occlusion as a viable alternative strategy to OACs for stroke prevention in AF, however, shortcomings in this approach remain. ${ }^{4,5}$ Chief among the limitations are the high frequency of periprocedural complications and question marks surrounding the clinical impact of residual peri-device flow over the long term. ${ }^{2}$ A separate and significant drawback is the fact that patients need to continue anticoagulation, which is not necessarily the case with epicardial devices. Recognised procedure- and device-related complications associated with catheter occluder options include pericardial effusion or perforation, periprocedural stroke, device embolisation, bleeding and access-related vascular complications. ${ }^{1,5}$

The Watchman ${ }^{\circledast}$ device (Boston Scientific, Marlborough, MA, US) successfully demonstrated both non-inferiority and superiority over warfarin in the PROTECT-AF (Watchman Left Atrial Appendage System for Embolic Protection in Patients With Atrial Fibrillation; NCT00129545) trial for the combined outcome of stroke, cardiovascular death or other embolism. ${ }^{8}$ Yet paradoxically, there remains a dearth of clinical evidence for Watchman and similar percutaneous devices in OAC-ineligible patients - the specific target group for interventional LAA occlusion according to ESC guidelines. In PROTECT-AF, eligibility for long-term warfarin anticoagulation was actually a specific 
inclusion criteria for the trial and $\sim 5 \%$ of patients were excluded at the screening stage due to inability to take warfarin. ${ }^{8}$ Patients treated with Watchman typically require a minimum of 6 weeks of post-procedural oral anticoagulation or dual antiplatelet therapy to facilitate endothelialisation of the device and reduce the risk of left atrial thrombi. ${ }^{3}$ Also, one consensus statement advises to continue single antiplatelet therapy indefinitely thereafter. Although the best strategy for post-procedural anticoagulation following Watchman placement is in flux, there is a trend away from using warfarin for 6 weeks. This presents a clear clinical dichotomy when considering percutaneous LAA occlusion devices in patients with true contraindications to OACs. ${ }^{3,4}$

The LAA has a complex and diverse anatomy with four broad morphological classifications - chicken wing (48\%), cactus (30\%), windsock (19\%) and cauliflower (3\%). ${ }^{2}$ Due to individual disparities in LAA morphology, patients can only be considered for a percutaneous LAA occlusion device if their cardiac anatomy is deemed suitable. ${ }^{4}$ A significant proportion will therefore be inherently ineligible for catheter closure due to anatomical limitations and underlying variability in their LAA morphology. Typically, the Watchman device can accommodate $95 \%$ of LAA anatomy but use may be restricted based on the maximum LAA ostium diameter, the LAA depth and the threshold of shoulder protrusion into the left atrium, among other considerations. ${ }^{10}$ Particularly challenging anatomies for placement of percutaneous devices include proximal and severely sharp-angled chicken wing configuration, certain cactus configuration and limited depths. ${ }^{10}$ Published series clearly illustrate how anatomy-based selection for occluder devices is required to accommodate patients' unique and individual anatomical variability. ${ }^{4}$ For example, of the 4,998 patients initially screened for inclusion in the PROTECT-AF trial of the Watchman device, 1,945 were excluded for 'various clinical and echocardiographic reasons' ${ }^{8}$

For patients not suitable for a percutaneous occluder approach, epicardial devices such as the Atriclip LAA Exclusion System (AtriCure, Mason, OH, US) present an important, alternative option. Atriclip is applicable to all comers irrespective of their LAA morphology and allows for immediate cessation of anticoagulation, making it a suitable choice for patients who are ineligible for catheter closure due to anatomical restrictions or for patients with contraindications to OACs. ${ }^{3.4}$ The Atriclip can be placed irrespective of the LAA anatomy or left atrial dilatation and is not restricted by ostial size."11 Epicardial devices also benefit from an absence of interface between intracardiac foreign body and blood, potentially reducing the risk of recognised percutaneous complications such as thrombosis and infection. In addition, embolism is not an issue with epicardial devices. ${ }^{3}$

\section{Atriclip clinical evidence}

The Atriclip is the most widely used LAA exclusion device and comprises a single-use, sterile, repositionable, self-closing clip which is pre-loaded onto a single-use clip applicator. ${ }^{3}$ The clip itself is made from two parallel titanium tubes with elastic nitinol springs covered by a knitted braided polyester. ${ }^{2,3}$ The delivery system allows for redeployment/repositioning of the device as required, thereby facilitating optimal placement of the clip at the base of the LAA, permanently and totally occluding the appendage from circulating blood in the left atrium. ${ }^{2,3}$

Although viewed as a 'surgical' approach, deployment of the Atriclip device is a relatively simple and short procedure associated with minimal complications. ${ }^{3}$ Exclusion of the LAA can be achieved without injury to the heart or surrounding structures during concomitant open cardiac surgery. ${ }^{1}$ The Atriclip can also be deployed as a stand-alone,
Figure 1: Computer tomography showing LAA (blue arrow in $A$ ) and stable clip position (blue arrow in B) with durable LAA occlusion with Atriclip after 3, 12 and 36 months
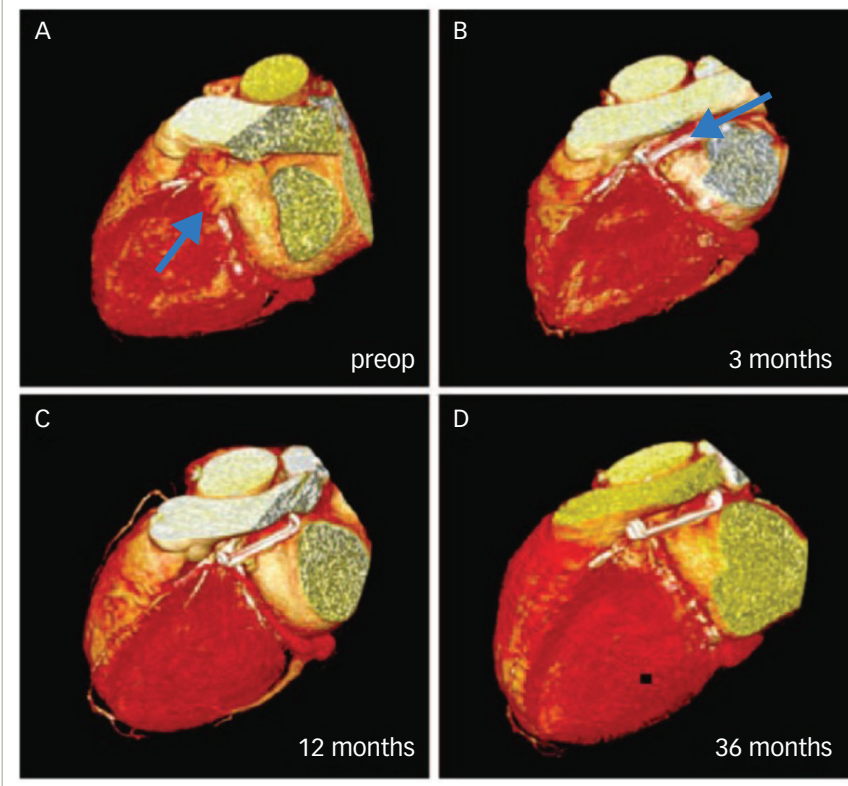

Reused with permission from Professor Sacha Salzberg.

minimally invasive thoracoscopic procedure. ${ }^{2,3,5}$ Placement of the clip is non-traumatic and does not entail puncture of the atrial septum, groin or pericardial membrane. However, Atriclip placement is typically limited to surgeons performing the procedure. From a cost perspective, Atriclip compares favourably to both other LAA occlusion devices and long-term OACs. ${ }^{3}$

Evidence from several clinical studies supports the safety profile, efficacy and durability of epicardial LAA occlusion using the Atriclip device. 1,4,5 Positive short-term safety and durability outcomes were attained with Atriclip in the prospective, non-randomised, multicentre EXCLUDE (Exclusion of Left Atrial Appendage with AtriClip Exclusion Device in Patients Undergoing Concomitant Cardiac Surgery; NCT00779857) trial.' Of the 71 patients undergoing cardiac surgery, 70 had successful placement of the Atriclip device and there were no reported device-related adverse events. The LAA occlusion rate confirmed by both computed tomography (CT) angiography and transoesophageal echocardiography (TEE) exceeded 98\%. ${ }^{1}$

The initial 'first in man' clinical study of Atriclip enrolled 40 patients with AF undergoing elective cardiac surgery and further validated the short-term feasibility and safety of epicardial LAA clipping. ${ }^{4}$ Clinical criteria excluding patients from receiving Atriclip in this trial included known LAA thrombus (evaluated by TEE), history of pericarditis, recent myocardial infarction and allergy to device components. ${ }^{4}$ Long-term data from this prospective device study after 3 years showed 100\% durability and complete LAA occlusion confirmed by CT imaging. ${ }^{4}$ In total, 36 patients were followed up for a mean duration of $3.5 \pm 0.5$ years. Clip position remained stable over this 3.5-year period with no secondary dislocation evident on CT (Figure 1). Intracardiac thrombi did not occur and none of the LAA became reperfused: LAA occlusion was total and complete in $100 \%$ of patients. No strokes were documented during follow-up of this trial and anticoagulation could often be discontinued. From a safety perspective, no device-related complications or mortality occurred. ${ }^{4}$ 
A further study examined outcomes, including stroke risk impact, in 291 patients fitted with the Atriclip during open-heart surgery - 40 from the prospective trial cohort ${ }^{4}$ and 251 from a consecutive institutional registry. ${ }^{5}$ This mixed surgical cohort also included patients with valvular $\mathrm{AF}$, in contrast to trials of Watchman that were carried out strictly in non-valvular AF. ${ }^{5}$ Atriclip delivery was performed in patients undergoing cardiac surgery (including isolated coronary artery bypass grafting, valve or combined procedures) with successful LAA occlusion controlled visually during the procedure and confirmed with simultaneous TEE, with subsequent repositioning as required. Long-term CT work-up in selected patients revealed LAA occlusion was $100 \%$ complete and durable 5 years or more post-implant, with no evidence of residual reperfusion or significant LAA stumps. In confirmation of the robust safety profile, no patients experienced device-related complications over the entire follow-up period post-implant, which ranged from 5.1-8.1 years. This study also provided the first circumstantial evidence of a significant reduction in stroke risk with Atriclip. The 166 patients who discontinued OACs after Atriclip deployment showed a relative stroke rate reduction of $87.5 \%$ $(0.5 / 100$ patient-years) compared with the expected rate for this $\mathrm{CHA}_{2} \mathrm{DS}_{2}$-VASC cohort (4.0/100 patient-years). ${ }^{5}$

As a stand-alone procedure, placement of the Atriclip device is not well recognised and available evidence remains limited. However, results from a recent case series of five patients ineligible for percutaneous treatment indicate that minimally invasive thoracoscopic closure of the LAA with Atriclip is a safe and feasible approach. In all patients, complete LAA closure was achieved without residual flow (confirmed by TEE) and with no complications or thrombi during 7.5 months of follow-up. ${ }^{11}$

Importantly, the Atriclip device not only completely excludes the LAA from the circulation but also affords complete electrical isolation, with no ongoing communication to the appendage..$^{1,4}$ As an occlusion technique, clipping may therefore offer adjunctive electrophysiological benefits given the arrhythmogenic role of the LAA.,12
From the available evidence, Atriclip appears to compare favourably with the Lariat catheter-based epicardial closure device, which is associated with a technical success rate of $93 \%$ and a lower procedural success of $83 \%$ due to major complications. ${ }^{10}$

Currently, Atriclip remains limited by a lack of supportive evidence from large-scale, prospective, randomised trials. Several studies are already underway to plug these data gaps, including a large randomised trial (NCT01561651) comparing surgical LAA occlusion with epicardial devices like Atriclip to no occlusion in over 4,500 patients with AF scheduled for routine cardiac surgery, plus a prospective, multicentre study (NCT01997905) of stand-alone Atriclip LAA occlusion for non-valvular AF. ${ }^{5,13,14}$ As well as adding to the existing evidence base for the safety and durability of Atriclip, conclusive clinical data from these studies could serve to strengthen the guidelines positioning of LAA closure - both epicardial and percutaneous - as a valid stroke-prevention strategy.

\section{Conclusions}

There is a clear need for further optimisation in LAA occlusion and adoption of a tailored approach that takes into account patient-specific anatomical and morphological variability, as well as any future need for anticoagulation. Electrophysiologists, as well as cardiac surgeons, together in the AF heart team, have a key role to play in delivering this tailored treatment strategy, ensuring the optimal device and technique is selected for each individual patient with AF to achieve the over-arching aim of safe, complete and durable LAA occlusion.

Catheter-based percutaneous approaches remain an important part of the electrophysiologists' toolkit for LAA occlusion but fail to deliver a $100 \%$ solution due to inherent limitations. Minimally invasive epicardial LAA clipping therefore represents an important alternative therapeutic option for patients with AF who are not amenable to catheter closure and/or anticoagulation. The Atriclip epicardial device is suitable for all patients irrespective of their LAA morphology and has demonstrated long-term safety, durable LAA occlusion and a significant reduction in stroke risk. $\square$
1. Ailawadi G, Gerdisch M, Harvey R, et al. Exclusion of the left atrial appendage with a novel device: early results of a multicenter trial. J Thorac Cardiovasc Surg. 2011;142: 1002-9.

2. Caliskan E, Cox J, Holmes D, et al. Interventional and surgical occlusion of the left atrial appendage. Nat Rev Cardiol. 2017; Epub ahead of print.

3. Ramlawi B, Abu Saleh W, Edgerton J. The left atrial appendage: target for stroke reduction in atrial fibrillation. Methodist Debakey Cardiovasc J. 2015;11:100-3.

4. Emmert M, Puippe G, Baumuller S, et al. Safe, effective and durable epicardial left atrial appendage clip occlusion in patients with atrial fibrillation undergoing cardiac surgery: first long-term results from a prospective device trial. Eur 」 Cardiothorac Surg. 2013;45:126-31.

Cardiothorac Surg. 2013;45:126-31.
5. Caliskan E, Sahin A, Yilmaz M, et al. Epicardial left atrial appendage Atriclip occlusion reduces the incidence of stroke in patients with atrial fibrillation undergoing cardiac surgery. Europace. 2018;20:e105-14

6. Kirchhof P, Benussi S, Kotecha D, et al. 2016 ESC guideline for the management of atrial fibrillation developed in collaboration with EACTS. Europace. 2016;18:1609-78.

7. Kanderian A, Gillinov AM Pettersson G, et al. Success of left atrial appendage closure: assessment by transesophageal echocardiography. J Am Coll Cardiol. 2008;59:924-9.

8. Reddy V, Sievert $H$, Halperin J, et al. Percutaneous left atrial appendage closure vs warfarin for atrial fibrillation: a randomized clinical trial. JAMA. 2014;312:1988-98.

9. Meier B, Blaauw $Y$, Khattab A, et al. EHRA/EAPCI consensus statement on catheter-based left atrial appendage occlusion. Statement on catheter-based left atria
Eurolntervention. 2015:10:1109-25.

10. Saw J, Lempereur M. Percutaneous left atrial appendage closure: procedural techniques and outcomes. JACC Cardiovasc Interv. 2014;7:1205-20.
11. Akca F, Verberkmoes $\mathrm{N}$, Verstraeten $\mathrm{S}$, et al. Is there an alternative treatment for patients intolerant to antiplatelet therapy if percutaneous left atrial appendage closure is considered? Neth Heart J. 2017;25:510-15.

12. Di Biase L, Burkhardt J, Mohanty P, et al. Left atrial appendage isolation in patients with longstanding persistent AF undergoing catheter ablation: the BELIEF trial. J Am Coll Cardiol. 2016;68:1929-40.

13. ClinicalTrials.gov. NCT01561651, Left Atrial Appendage Occlusion Study III (LAAOS III). Available at: https://clinicaltrials.gov/ct2/show/ NCT01561651?term =NCT01561651\&rank=1 (accessed 17 January 2018).

14. ClinicalTrials.gov. NCT01997905, Stroke Feasibility Study. Available at: https://clinicaltrials.gov/ct2/show/ NCT01997905? term =NCT01997905\&rank=1 (accessed 17 January 2018). 